\title{
¿Es el cristianismo responsable de la crisis ambiental del planeta?
}

\section{Is Christianity responsible for earth's environmental crisis?}

\author{
Emilio Chuvieco Salinero*
}

\section{INTRODUCCIÓN ${ }^{1}$}

Resulta casi superfluo tener que demostrar que los problemas ambientales tienen una gran repercusión en la sociedad contemporánea, manifestada tanto en la atención que se les dedica en los medios de comunicación, como en las políticas públicas o las actividades empresariales. Los problemas ambientales están también muy presentes en la investigación científica, incluyendo una amplia variedad de cuestiones y de metodologías de análisis: desde el descubrimiento de nuevas fuentes de energía, hasta las técnicas para hacerlas más eficientes, desarrollar medios de producción menos contaminantes, mejorar la gestión de residuos, o aplicar técnicas más eficientes de restauración.

Una de las líneas de trabajo más relacionadas con el ámbito geográfico es la que se centra en el análisis de las causas y las consecuencias del impacto humano sobre el medio ambiente. Por encima de la importancia fundamental de determinados procesos naturales, se ha llegado a un cierto consenso internacional sobre el papel protagonista de las actividades humanas en algunos de los

\footnotetext{
*Departamento de Geografía, Universidad de Alcalá (emilio.chuvieco@uah.es).

${ }^{1}$ Parte de este trabajo se presentó en el simposio internacional: Ciencia y Religión en el siglo XXI: diálogo o confrontación, organizado por la Fundación Areces en Noviembre de 2011 (Chuvieco, 2012a). Para una visión más extensa sobre esta problemática puede también consultarse Chuvieco y Martin (2012).
} 
problemas ambientales más relevantes, singularmente en los derivados del cambio climático, la pérdida de biodiversidad o la degradación del agua y del suelo.

Si los problemas tienen un origen humano, las soluciones pasan también por un cambio social, en buena parte asociado a cómo nos relacionamos con el planeta. Se hace más evidente la importancia de reorientar nuestro modelo de desarrollo, orientándolo hacia una utilización más armónica de los recursos, que garantice su equilibrio a largo plazo. En este marco, las cuestiones ambientales conectan directamente con nuestros hábitos de consumo, y, más en profundidad, con nuestros valores éticos y morales. La visión que cada uno tenga del ser humano y de su posición en el cosmos marcará el alcance de esas decisiones. Las posturas pueden ser tan variadas como las posiciones antropológicas que las avalen. En un extremo, asumir que el hombre tiene capacidad absoluta de utilizar los recursos a conveniencia; en el contrario, considerar que lo mejor que podemos hacer por el medio ambiente es autodestruirnos como especie, ya que el hombre es el principal cáncer del planeta.

La orientación de los individuos o de la sociedad hacia una u otra posición antropológica está notablemente influida por sus tradiciones culturales, entre las cuales un componente clave es la visión religiosa. Por esta razón, me parece de gran interés estudiar qué implicaciones tienen las grandes religiones sobre nuestras actitudes ante la conservación ambiental. Las religiones suponen planteamientos cosmológicos sobre la posición del hombre en relación con Dios, con los otros hombres y con el medio que le rodea. De esos planteamientos se deducen unos principios éticos y morales determinados, que explicarán —al menos parcialmente- unas determinadas conductas. En ambos casos, cualquier concepción religiosa lleva consigo determinadas implicaciones ambientales, ya que un planteamiento cosmológico debería implicar formas de hacer concretas, asumiendo lógicamente que los creyentes sean consecuentes con sus creencias. Esta relación fue subrayada por los promotores de una serie de seminarios sobre religión y ecología que promovió la Universidad de Harvard a inicios de la pasada década (Foltz et al., 2003; Hessel y Ruether, 2000; Tirosh-Samuelson, 2002; Tucker y Williams, 1997). En el prólogo de uno de los libros publicados con ocasión de esos seminarios, se señalaba que las «... religiones proporcionan historias interpretativas sobre quiénes somos, qué es la naturaleza, de dónde venimos y a dónde vamos. Esto comprende una visión global de la sociedad. Las religiones también sugieren cómo deberíamos tratar a otros seres humanos y cómo deberíamos relacionarnos con la naturaleza. Esos valores configuran las orientaciones éticas de la sociedad. En consecuencia, las religiones generan visiones del mundo y normas éticas que están en el fondo de las actitudes 
fundamentales y de los valores de las diferentes culturas y sociedades» (Tucker y Grim, 2003, p. xvi).

Por ejemplo, si un creyente considera que la naturaleza es sagrada, su actitud ante ella será completamente distinta a si piensa que Dios le ha dado la naturaleza para su uso absoluto. Por eso, el análisis de las relaciones entre religión y conservación ambiental debería revisar cuál es la visión de la naturaleza que emana de los principios en una determinada religión, qué indica sobre el papel del hombre en la Creación, sobre sus relaciones con las demás criaturas, o en qué medida le hace responsable ante Dios del uso de los recursos terrestres. Si existe una consistencia ética con esos planteamientos cosmológicos, adoptar uno u otro enfoque debería llevar consigo diferencias significativas en las relaciones prácticas de los seres humanos con el medio ambiente que les rodea.

En los años sesenta se publicó uno de los primeros trabajos que señalaba la conexión entre religión y conservación ambiental, en este caso indicando la relación entre la visión cosmológica que favorecía el cristianismo y el uso abusivo que la civilización occidental ha hecho de los recursos terrestres (White, 1967). Desde entonces, se ha especulado mucho sobre la fundamentación religiosa de la explotación de la naturaleza, y el enfoque más o menos conservacionista de las grandes religiones. En este trabajo, pretendemos mostrar, en una primera parte, algunos argumentos que discuten en el plano teórico si el cristianismo es o no menos conservacionista que otras religiones. En la segunda parte, llevamos esa discusión a un terreno más práctico analizando las diferencias en distintos indicadores ambientales para países con distintas tradiciones religiosas. Se considera que si el cristianismo fuera menos amigable con el entorno, los países de tradición cristiana deberían presentar indicadores ambientales significativamente peores que los encontrados para otros con distintas tradiciones religiosas, lógicamente excluyendo en la medida de lo posible otros factores de control. Si esa hipótesis no se verifica, deberíamos concluir que, en el plano práctico, tampoco podría considerarse al cristianismo como una religión más agresiva con el entorno que otras.

\section{ORIGEN DE LAS CRÍTICAS AMBIENTALES AL CRISTIANISMO}

Para algunos planteamientos ecocéntricos, el origen de la crisis ambiental está en una actitud arrogante del ser humano respecto a las demás especies, que lleva a utilizarlas en su exclusivo provecho (Berry, 1988). Para algunos pensadores, esta arrogancia del hombre tiene un origen religioso: obedece 
principalmente a la cosmología judeo-cristiana, que confiere al ser humano un papel de dominio, donde los demás seres creados no tienen utilidad en sí mismos, sino en la medida en que son útiles para servir a nuestras necesidades.

En el origen de esta hipótesis está el artículo que el historiador Lynn White Jr., publicó en 1967 en la revista Science, con motivo de su intervención ante la asociación americana para el avance de la ciencia (White, 1967). En este trabajo se indicaban dos elementos que, a juicio del autor, justifican una relación directa entre cristianismo y crisis ecológica. Por un lado, el gran avance de la ciencia y la capacidad de transformar el territorio que se produce en la Europa cristiana y, por otro, la concepción antropológica particular del cristianismo, que pone al ser humano en el centro de la Creación, por encima de las demás criaturas.

En cuanto al primer elemento, diversos autores han subrayado la conexión histórica entre el origen de la ciencia y el cristianismo, basándose en la propuesta que realiza de un Dios «razonable», que diseña el Universo con unas reglas estables, inteligibles (Woods, 2005). Este principio, unido al impulso académico que supuso la creación de las primeras universidades en los siglos XII y XIII, permitió la eclosión en los siglos XVI y XVII de una verdadera revolución científica, que se da únicamente en países del occidente cristiano y por científicos de convicciones cristianas —Copérnico, Galileo, Newton, Descartes, Kepler, Pascal- A ello se une el desarrollo técnico que había permitido las mejoras en la producción agraria, una incipiente industria artesana y una navegación cada vez más sólida. Es principalmente en el siglo XvII cuando convergen el desarrollo científico y la tecnología, lo que brinda al ser humano una capacidad de transformación del medio natural sin precedentes.

El segundo factor que relaciona el cristianismo con la crisis ecológica, según White, procede de la visión antropológica que propone la Biblia, marcada por una clara pre-eminencia del ser humano sobre las demás criaturas, al ser el único ser creado a imagen y semejanza de Dios. Se refiere de modo particular White a un párrafo del primer capítulo del Génesis: «Sed fecundos y multiplicaos y henchid la tierra y sometedla; mandad en los peces del mar y en las aves de los cielos y en todo animal que serpea sobre la tierra. Dijo Dios: "Ved que os he dado toda hierba de semilla que existe sobre la haz de toda la tierra, así como todo árbol que lleva fruto de semilla; para vosotros será de alimento. $\mathrm{Y}$ a todo animal terrestre, y a toda ave de los cielos y a toda sierpe de sobre la tierra, animada de vida, toda la hierba verde les doy de alimento" Y así fue» (Génesis, 1: 28-30).

Esa visión cosmológica de la tradición judeo-cristiana habría llevado consigo — según White - una relación de completa subordinación de la natura- 
leza respecto al ser humano, que resulta única respecto a otras tradiciones religiosas: «El cristianismo, en contraste absoluto con el paganismo antiguo y las religiones de Asia (a excepción del Zoroastrianismo), no solo estableció un dualismo entre la naturaleza y el hombre, sino también insistió en que es voluntad de Dios que el hombre explote la naturaleza para sus propios fines» (White, 1967, p. 1205). Además, la progresiva sustitución del paganismo por el cristianismo supuso un abandono de la consideración sagrada de la naturaleza, que se considera en el cristianismo una forma de idolatría.

En suma, para White, el cristianismo separa al hombre de la naturaleza y le hace considerarse por encima de las demás criaturas, considerándolas únicamente para su propia utilidad. En contraste con esta tradición dominante en el cristianismo occidental, resalta White la figura de San Francisco de Asís, que en su opinión supone un ejemplo insólito de acercamiento al mundo natural, llevado por una humildad cósmica que pone al hombre en un plano de igualdad con las demás criaturas creadas. El planteamiento de San Francisco resulta tan innovador frente a la concepción antropológica del cristianismo en ese tiempo que, siempre según la interpretación de White, sorprende que su mensaje no fuera rechazado por la jerarquía católica: «El primer milagro de San Francisco es el hecho de que no acabara sus días en la hoguera, como posteriormente acabarían muchos de sus seguidores más radicales. Fue tan claramente herético que un General de la orden franciscana, San Buenaventura, un gran y bondadoso cristiano, intentó suprimir los primeros vestigios del franciscanismo» (White, 1967, p. 1206).

Puesto que ese planteamiento religioso dominador de la naturaleza está en la raíz del desequilibrio del planeta, White propone cambiar el paradigma cultural, de origen cristiano, re-enfocando nuestro protagonismo en la relación con el medio natural que nos rodea. «Puesto que las raíces de nuestro problema son principalmente religiosas, el remedio debe también ser esencialmente religioso, lo llamemos así o no» (White, 1967, p. 1207). No llega a recomendar este autor la repudia del cristianismo a favor de otras religiones, sino más bien una reflexión que permita alterar la visión antropocéntrica a favor de una concepción más equilibrada con el medio, siguiendo precisamente la propuesta de San Francisco, a quien propone como patrón de los ecologistas.

Hasta ahí la postura de Lynn White, que ha servido para estimular un debate de enorme impacto. Su artículo aparece citado en una gran cantidad de trabajos académicos — casi 2.000, según mis últimas consultas—, que tienden a sentar al cristianismo en el banquillo de los acusados. Otros autores han continuado esta línea de pensamiento en publicaciones más recientes — por 
ejemplo Berry en 1988 y varios autores de la obra colectiva editada por Berry en 2006- Me parece interesante reseñar que estas alegaciones se realizan sobre algunas características del cristianismo que, hasta hace pocas décadas, se han considerado como síntomas de progreso, como es el caso de la superación del paganismo - que se consideraba un modo de superstición, de adoración de elementos materiales que prevenía el desarrollo científico-, del humanismo que propone - el hombre es el centro de la Creación-, o de su apuesta por la racionalidad del conocimiento - que permite alumbrar la ciencia moderna-.

\section{LA RESPUESTA A LAS CRÍTICAS: ARGUMENTOS ESCRITURÍSTICOS}

Indudablemente el texto de White era provocador y, en consecuencia, provocó la reacción de numerosos académicos, que contestaron al texto desde diversas disciplinas, enriqueciendo, gracias a ello, la orientación ambiental del cristianismo. La defensa de las acusaciones lanzadas por White puede resumirse en dos bloques: por un lado, se intenta esclarecer el sentido genuino del mensaje bíblico sobre las relaciones entre el hombre y el resto de la Creación, y por otro se pretende demostrar que existe una tradición cristiana de conservación ambiental que no se limita a la figura de San Francisco. Trataremos estos dos argumentos por separado.

Ya hemos visto cómo en el primer libro de la Biblia se concede al ser humano una posición de dominio sobre el resto de la Creación. Ahora bien, conviene interpretar ese pasaje en un contexto más amplio, conectándolo con otros pasajes de la Biblia, donde se matiza notablemente este supuesto papel dominador. Por ejemplo, en el segundo capítulo del Génesis se presenta al ser humano como parte de la misma tierra que es llamado a preservar: «Entonces Yahveh Dios formó al hombre con polvo del suelo, e insufló en sus narices aliento de vida, y resultó el hombre un ser viviente» (Génesis 2, 4-7). El primer capítulo del Génesis proviene de una tradición que se denomina Yavista, mientras el segundo procede de la llamada tradición sacerdotal, unos 500 años más antigua. En este segundo relato se presenta al hombre como fruto de la tierra, extraído de la misma materia a la que pertenecen el resto de las criaturas, lo que marca de alguna manera su fragilidad — «Porque eres polvo y al polvo tornarás»- (Gen 3: 19). El nombre de Adam, viene de Adamah, que significa suelo, tierra. El ser humano es de la misma condición que el resto de la Creación, aunque ciertamente el texto sagrado indica que es fruto de una especial intervención de Dios, que le insufla aliento de vida. 
Volviendo al primer capítulo del Génesis, también conviene tener en cuenta que el término «dominio», con el que aparece ese encargo de Dios sobre la creación material, no debe considerarse como una licencia absoluta, sino más bien como una administración delegada. En pocas palabras, la Naturaleza no fue donada al hombre por el Creador como un patrimonio despótico, sino más bien confiada a su cuidado, lo que implica su utilización responsable. Esta interpretación sería más acorde con el segundo capítulo del Génesis, cuando se indica que «...tomó, pues, Yahveh Dios al hombre y le dejó en el jardín de Edén, para que lo labrase y cuidase» (Gen, 2, p. 15). En resumen, la imagen del Edén no es la de un coto de caza, ni una finca, sino más bien la de un jardín que los hombres reciben de Dios para cuidarlo cabalmente. No se trata, además, de un territorio propio, sino de un encargo de Dios, que nos ha creado, no lo olvidemos, de la misma materia (suelo-tierra) que nos circunda.

La tesis de White no tiene en cuenta un principio básico de la exégesis cristiana: que la interpretación de un pasaje debe hacerse a la luz de otros, siguiendo su concordancia global y su contexto histórico. Extraer del texto de Génesis 1 un concepto de dominio absoluto del hombre sobre las demás criaturas, supone negar otros muchos pasajes de la Sagrada Escritura, que muestran cómo Dios es el propietario último de todo lo que existe y que el uso que haga el hombre de otros seres se basa en un papel subsidiario. Incluso el dominio real, en la tradición judía, se considera delegado de Dios, ya que los reyes son elegidos y ungidos por los profetas, particularmente en el caso de Saúl y David, por lo que tienen que dar cuenta de su dominio (Harrison, 2006). En consecuencia, la palabra dominio no indica un poder absoluto en la terminología bíblica, sino más bien un encargo divino; aplicado a las demás criaturas de la Creación. Por tanto, Génesis 1 concede al hombre un papel protagonista, pero subsidiario al fin y al cabo, que implica dar cuentas ante Dios del uso que hagamos de los recursos terrestres. En resumen, el significado de «dominad la tierra» indica un dominio vicario, que demanda una responsabilidad de quien lo ejerce ante quien lo encarga.

En esta interpretación de la Biblia se ancla el concepto de «administración o mayordomía ambiental» (environmental stewardship), que se ha desarrollado bastante en las últimas décadas, tanto en el marco de la teología protestante como, más recientemente, de la católica. El término fue utilizado por vez primera por Matthew Hale en el siglo XVII, pero ha tenido un gran auge en los últimos años (Berry, 2006). Parte de la base que los recursos de la Creación no pertenecen al hombre, sino que le son cedidos — delegados- por Dios. En la medida en que no son nuestros, somos responsables y Dios nos pedirá cuenta 
del uso que hagamos de ellos ante el mismo Creador, que es quien nos los confía (Houghton, 2006).

La imagen bíblica del administrador de recursos ajenos proviene de algunas parábolas del Evangelio, como la del administrador infiel (Lc, 16), el señor que entrega talentos a sus empleados para que negocien en su ausencia (Mt, 25: 14-28), o el arrendatario de la viña que tiene que dar los frutos a su tiempo (Mt, 21: 33-41; Lc 13: 6-9).

El valor intrínseco que los textos bíblicos conceden a los seres creados, más allá de servir a las necesidades humanas, puede también comprobarse en diversos pasajes, que recogen con claridad el sentido de alabanza a Dios que todas las criaturas profesan. Este sería el caso del "Himno de los tres jóvenes", extraído del libro del profeta Daniel $(3,57-88)$, que muestra de manera muy plástica cómo la adoración a Dios no se restringe al hombre, sino que alcanza a todo el universo creado: «Aguas, bendecid al Señor; ejércitos del Señor, bendecid al Señor. Sol y luna, bendecid al Señor; astros del cielo, bendecid al Señor. Lluvia y rocío, bendecid al Señor; vientos todos, bendecid al Señor. Fuego y calor, bendecid al Señor; fríos y heladas, bendecid al Señor. Rocíos y nevadas, bendecid al Señor; témpanos y hielos, bendecid al Señor...». Este texto es especialmente relevante, ya que forma parte de la oración oficial de la Iglesia - liturgia de las horas- desde tiempos muy antiguos.

Para algunos autores, aunque este concepto supone una interpretación más benigna que una visión antropocéntrica del cristianismo, no resuelve el problema ambiental, porque sigue tratándose de un planteamiento equivocado de las relaciones con el medio natural. Por ejemplo, para Clare Palmer (2006) el concepto de administración ambiental está asociado a la idea medieval de dependencia feudal: el siervo recibe el encargo del señor, que es dueño absoluto de los bienes sobre los que delega. Además de que esa figura implica un régimen social ya superado, que tiene connotaciones despóticas poco aceptables actualmente, Palmer se pregunta, ¿en nombre de quién ejercemos esa administración? El concepto está en el Nuevo Testamento, como hemos indicado, pero no de modo explícito aplicado al cuidado de la Tierra. Por otro lado, administrar unos bienes requiere capacidad de hacerlo, pero no está claro que tengamos conocimiento suficiente del sistema terrestre para hacerlo con propiedad, ni tampoco que esa administración sea necesaria para el buen funcionamiento del planeta, ya que de hecho la Tierra ha estado funcionando durante muchos millones de años sin nuestro concurso, y no está claro que nuestra intervención la haya perfeccionado siempre. Desde un punto de vista más teológico, Palmer indica que el concepto de administración podría indicar que Dios está ausente del mundo, como si Él no fuera capaz de ejercer ese 
dominio, lo que pondría en tela de juicio la providencia divina sobre todas las cosas. En esta misma línea, otros autores opinan que la idea de administración ambiental implica la imagen de Dios como un terrateniente absentista, que anula la espontaneidad del hombre para vivir en la Tierra.

Los partidarios del concepto de administración ambiental no consideran que estas críticas sean suficientemente sólidas, ya que la figura del dominio delegado está presente en muchos párrafos de la Biblia, que refuerzan la única propiedad de Dios sobre la Creación. Por ejemplo, en el Levítico leemos la legislación que propone el texto sagrado sobre la redención de la tierra en los periodos sabáticos, pues «...la tierra no puede venderse para siempre, porque la tierra es mía, ya que vosotros sois para mí como forasteros y huéspedes» (Lev 25: 23). Por otro lado, la «cesión para el uso» que Dios nos realiza de la Creación no implica un abandono de su providencia ordinaria, ni una eliminación de la libertad humana, como no desaparece la libertad del arrendatario de una propiedad o del director de un fondo de inversiones; ambos manejan recursos ajenos, y en esa medida tienen que entregar un balance de su gestión, pero según su propia decisión para hacerlos rendir de la manera más adecuada. Por tanto, el concepto de administración delegada de Dios nos llevaría a ser más responsables en el uso de los recursos del planeta, orientándolos a satisfacer nuestras necesidades vitales o las que nos engrandecen como personas, en lugar de hacerlo despótica o caprichosamente.

\section{LA RESPUESTA A LAS CRÍTICAS: TRADICIÓN HISTÓRICA}

Junto a esta tradición de «dominio delegado», evidentemente más benigna para la conservación ambiental que la del dominio absoluto criticada por White, es interesante apuntar que en el cristianismo se han dado históricamente otras tradiciones de acercamiento a la naturaleza, en buena parte similares a las que pueden detectarse en otras grandes religiones (Chuvieco, 2012b).

La monografía de Sorrell sobre los aspectos ambientales de la figura de San Francisco (Sorrell, 1988), disiente con la hipótesis de White de que se trate de una figura aislada en el contexto de la tradición cristiana. Para este autor, el santo de Asís recoge una tradición eremita muy arraigada, que encontró en el contacto con la naturaleza su vehículo de encuentro con Dios; a la vez que entronca con la tradición poética clásica, de Ovidio a Virgilio, que se expande en la Edad Media en los cantos de trovador, que San Francisco conocía bien en sus años más jóvenes. También hay una cierta tradición científica, recogida 
en las compilaciones del saber clásico que realizan San Isidoro, San Beda o Rabano Mauro que ponen en la observación de la naturaleza un acento muy característico. Finalmente, San Francisco aprovecha también una tradición comunitaria de los primeros monasterios benedictinos y cistercienses, que mostraron una actitud contemplativa y activa ante la naturaleza.

Algunos testimonios de esta tradición cristiana de respeto y admiración por la naturaleza aparecen citados en la misma obra de Sorrell. Por ejemplo, San Agustín, muy conectado con los poetas clásicos, que conocía bien, da rienda a su sentido estético en uno de los últimos capítulos de La Ciudad de Dios: "Como me referiré al resto de la creación, con toda su belleza y utilidad de que la divina liberalidad ha hecho merced al hombre, aunque postrado y condenado a tantos trabajos y miserias, para que la goce y se aproveche de ella, ¿con qué palabras la referiremos? ¿Qué diré de la belleza, tan grande y tan diversa, del cielo, de la tierra y del mar; de una abundancia tan grande y de la hermosura tan admirable de la misma luz en el sol, la luna y las estrellas...» (San Agustin, pp. 413-426).

Otros testimonios en línea similar pueden recogerse en las vidas de San Martín de Tours, de San Columbano, o de San Basilio. Los primeros monjes benedictinos valoran notablemente el trabajo manual, como complemento a la oración, lo que les lleva a un mayor contacto con la naturaleza y a proponer un cierto sentido de lo que hoy llamaríamos sostenibilidad. La reforma cisterciense, ya en el siglo XII muestra también diversos elementos comunes con el mensaje franciscano, en su apreciación por los lugares bellos, y en su sentido de la alabanza a Dios de todas las criaturas. Hay muchos escritos en esta línea, mereciendo destacarse "La descripción de Clairvaux", de gran belleza literaria. En otro escrito, en forma de diálogo, uno de los primeros cistercienses, Idung de Prüfening (1153-1174), incluye la siguiente admonición entre dos monjes: «Eres un teólogo muy simple si no reconoces que las criaturas inanimadas, desprovistas de sentido y vida, hablan a Dios y dan gloria a Dios. ¿Cuál si no sería el sentido del Himno de los jóvenes y de las alabanzas de los salmos, donde todas las criaturas están invitadas a alabar a Dios?» (Sorrell, 1988, p. 31).

También resulta destacada la figura de Santa Hildegarda de Bingen (10981178), benedictina alemana, mujer de gran cultura, que tuvo un notable impacto en su tiempo. Entre sus obras poéticas muestra también un notable aprecio por la belleza de la creación: «Como el Creador ama a su creación, así la creación ama al Creador. La creación, naturalmente, fue diseñada para ser adornada, ser mostrada, ser regalada con el amor del Creador. El mundo entero ha sido abrazado por este beso» (citado por McDonagh, 1986, p. 135). 
Sobre esta tradición, la figura de San Francisco de Asís (1182-1226) emerge de una manera muy particular, no sólo por su valoración de la naturaleza, sino también por otros aspectos de su espiritualidad, lo que le hace merecer un papel central en la historia del cristianismo. En lo que se refiere a su valoración de la naturaleza, el enfoque espiritual de San Francisco ciertamente supone un salto cualitativo sobre la tradición precedente. A partir de una vida de profunda piedad, una tradición eremita que gusta de la austeridad y la soledad, su vida denota múltiples manifestaciones de una extraordinaria unión y afecto por todas las criaturas. Sus biógrafos interpretan esa cercanía con los animales silvestres como una manifestación de su inocencia y santidad de vida, como un reflejo de una armonía que se remonta al estado previo al pecado original. Su sermón a los pájaros marca un momento singular en su vida, una cierta conversión interior, que supone extender a todas las criaturas su vocación apostólica: en suma, incluirlas también en la predicación evangélica, como manifestación de afecto familiar, de cuidado y de servicio. La actitud más profunda de San Francisco es la de un misticismo natural, que le lleva a conectar directamente con Dios en la contemplación de las criaturas, a trascender al Creador desde lo creado. Esa actitud interior se refleja extraordinariamente en El canto del hermano Sol, uno de los poemas más bellos de la literatura medieval: «Loado seas, mi Señor, con todas tus criaturas, especialmente el señor hermano sol, el cual es día, y por el cual nos alumbras. Y él es bello y radiante con gran esplendor, de ti, Altísimo, lleva significación. Loado seas, mi Señor, por la hermana luna y las estrellas, en el cielo las has formado luminosas y preciosas y bellas. Loado seas, mi Señor, por el hermano viento, y por el aire y el nublado y el sereno y todo tiempo, por el cual a tus criaturas das sustento (...)» (San Francisco, p. 1225).

Diversos autores han discutido el sentido último que tiene en el cántico la preposición «por», que utiliza San Francisco en las primeras estrofas. Para algunos, el «por» podría asimilarse a «mediante» —esto es, que Dios sea alabado por las criaturas-, lo que indicaría el deseo del santo de que los diversos elementos de la naturaleza den gloria a Dios. Siguiendo esa interpretación, este texto no sería muy distinto de otros que hemos citado previamente, como "El canto de los tres jóvenes" recogido en el libro del profeta Daniel. Un segundo sentido, mucho más innovador, da al «por» el significado de «a causa de», lo que supondría que el santo está pidiendo a los hombres que alaben a Dios ante la maravilla de los seres que ha creado. Este segundo sentido, muy acorde con diversos pasajes de la vida del Santo, propone que el hombre se goce en la Creación y que valore los beneficios que de ella recibe. En definitiva, se muestra una valoración original de la Creación, 
se pide a la humanidad que se alegre por ella y, de modo implícito, que la valore y la cuide.

En este segundo significado, el cántico refleja perfectamente el mensaje franciscano, que no ha de considerarse lógicamente como un alegato ecológico —estamos en el siglo XIII, no lo olvidemos-, sino como una valoración novedosa de la Creación, que acompaña al hombre en su oración, pero que también le ayuda a elevar su mente a Dios, como manifestación de la bondad y belleza divinas. Nos alejamos entonces de esta visión maniquea, que podría haber afectado a algunos autores católicos, pero que se muestra con más claridad en otros movimientos cristianos que fueron considerados heréticos por la Iglesia, como es el caso de los cátaros, contemporáneos de San Francisco, que recogiendo la tradición gnóstica y maniquea consideraban precisamente lo material como repudiable y abogaban por una ascética espiritualista, alejada de contaminaciones materiales. Para San Francisco, el orden material también es parte del plan divino de la Creación y, por tanto, no sólo no debería alejarnos de Dios, sino más bien debería acercarnos a su Presencia, elevándonos desde la contemplación de las criaturas a las perfecciones de su Creador.

Que el planteamiento antropocéntrico de occidente no se alterara sustancialmente, no implica que el mensaje de San Francisco fuera considerado sospechoso de herejía, como indica White en su artículo. En ningún momento de su vida se puso en duda la ortodoxia de San Francisco, que fue canonizado en un tiempo récord, apenas dos años después de su muerte. Tampoco es cierto que San Buenaventura mutilara los aspectos más controvertidos de su biografía, ya que su obra sobre el santo es sustancialmente la misma que tenemos de otras fuentes realmente solventes, como han mostrado los expertos más profundos de su obra. De hecho, «ningún biógrafo católico, ya fuera más temprano o más tarde, ha considerado heterodoxa la interpretación franciscana del medioambiente. Más aún, en las discusiones sobre asuntos de la orden franciscana que se tuvieron en el siglo XIII, con consejeros del Papa o con los propios Papas, ese tema nunca salió a colación» (Sorrell, 1988).

\section{EL LEGADO AMBIENTAL DEL CRISTIANISMO}

Una segunda línea de argumentación en respuesta a las críticas al cristianismo vendría dada por observar la situación ambiental de países con mayoría cristiana, respecto a otros donde son dominantes otras tradiciones religiosas. Si el cristianismo ha contribuido al deterioro ambiental, esperaríamos encontrar indicadores ambientales significativamente peores. Lógicamente para que 
esa comparación sea sólida es preciso que se controlen otros factores que influyen también en los indicadores ambientales, como son el nivel de desarrollo, o el nivel de gobernanza de los países. Además, es preciso comparar países que tengan un elevado grado de uniformidad religiosa, como para asumir que esa determinada religión realmente tiene un peso social significativo. Finalmente, tampoco es trivial medir el estado de salud ambiental de un país. Diversos organismos internacionales han propuesto sistemas basados en variables -indicadores - que pueden recolectarse de modo sistemático (Sotelo et al., 2011), pero aún no existe un indicador estándar que mida la situación ambiental de un territorio.

Conscientes de esas limitaciones, presentamos aquí un primer ensayo que evalúa la situación ambiental de los países en función de su tradición religiosa mayoritaria de cara a analizar si se evidenciaban diferencias significativas entre ellos. Las fuentes de nuestro análisis son dos:

1) Para el nivel de creencia religiosa, se han tomado las fuentes sociológicas más aceptadas, que coinciden con la percepción común sobre la preeminencia religiosa de cada país. Los datos cuantitativos se han tomado de una amplia cantidad de fuentes, principalmente de la CIA World Factbook, el Departamento de Estado de EE.UU., la encuesta Gallup y la de la Pew Foundation ${ }^{2}$. Para el análisis comparativo hemos seleccionado únicamente los países con una religión mayoritaria de acuerdo con los datos para 2007, entendiendo por ello los países en los que más del 65\% de su población se identifica con esa tradición. La tabla 1 incluye la relación de los países elegidos. Los que no alcanzaron el umbral indicado, se han recogido en la categoría Otros.

2) En cuanto al nivel de conservación ambiental de los países, he utilizado un índice de calidad ambiental denominado «Environmental Performance Index» (EPI) que es elaborado por el Center for Environmental Law \& Policy de la Universidad de Yale desde $2006^{3}$, en colaboración con el Center for International Earth Science Information Network (CIESIN) de la Universidad de Columbia, el World Economic Forum y el Joint Research Centre de la Unión Europea. Incluye 25 indicadores generados para 163 países, tomados de las fuentes más fidedignas posibles (Emerson et al., 2010). El índice global, que se mide en una escala

2 Todas ellas recogidas en: http://en.wikipedia.org/wiki/Religions_by_country (Fecha de consulta: abril 2012).

${ }^{3}$ Empleamos aquí la edición de 2010 que se encuentra disponible en: http://epi.yale.edu/ (Fecha de consulta: agosto 2012). 
Tabla 1

CLASIFICACIÓN DE PAÍSES POR SU RELIGIÓN MAYORITARIA

\begin{tabular}{|c|c|}
\hline Religión & Países \\
\hline Budismo & $\begin{array}{l}\text { Bhutan, Cambodia, Japón, Laos, Myanmar, Singapur, Tailan- } \\
\text { dia, Vietnam }\end{array}$ \\
\hline Cristianismo & $\begin{array}{l}\text { Alemania, Angola, Antigua and Barbuda, Argentina, Armenia, } \\
\text { Australia, Austria, Belice, Bielorrusia, Bolivia, Botsuana, Brasil, } \\
\text { Bulgaria, Burundi, Canadá, Republica centroafricana, Chile, } \\
\text { Colombia, Congo, Costa Rica, Croacia, Cuba, Chipre, Rep. } \\
\text { Dem. Congo, República dominicana, Ecuador, EE.UU., El Sal- } \\
\text { vador, Eslovaquia, Eslovenia, España, Filipinas, Guinea Ecua- } \\
\text { torial, Gabón, Georgia, Ghana, Grecia, Guatemala, Guyana, } \\
\text { Haití, Honduras, Hungría, Islandia, Irlanda, Italia, Jamaica, } \\
\text { Kenia, Letonia, Lituania, Luxemburgo, Macedonia, Malawi, } \\
\text { Malta, México, Moldavia, Namibia, Nicaragua, Noruega, Pa- } \\
\text { namá, Papúa Nueva Guinea, Paraguay, Perú, Polonia, Portu- } \\
\text { gal, Reino Unido, Rumania, Ruanda, Islas Salomón, Sur } \\
\text { África, Suazilandia, Suecia, Uganda, Ucrania, Uruguay, Vene- } \\
\text { zuela, Zambia, Zimbabue. }\end{array}$ \\
\hline Hinduismo & India, Nepal, Sri Lanka \\
\hline Judaísmo & Israel \\
\hline Islam & $\begin{array}{l}\text { Albania, Argelia, Azerbaiyán, Bahréin, Bangladesh, Brunei, Da- } \\
\text { russalam, Djibouti, Egipto, Gambia, Guinea, Indonesia, Irán, } \\
\text { Iraq, Jordán, Kuwait, Kirguistán, Libia, Maldivas, Mali, Mauri- } \\
\text { tania, Marruecos, Níger, Omán, Pakistán, Qatar, Arabia Saudí, } \\
\text { Senegal, Sudán, Siria, Tayikistán, Túnez, Turquía, Turkmenis- } \\
\text { tán, Uzbekistán, Yemen }\end{array}$ \\
\hline Otros & $\begin{array}{l}\text { Bélgica, Benín, Bosnia y Herzegovina, Burkina Faso, Camerún, } \\
\text { Chad, China, Corea del Norte, Corea del Sur, Costa de Marfil, } \\
\text { República Checa, Dinamarca, Eritrea, Estonia, Etiopía, Fiji, } \\
\text { Finlandia, Francia, Guinea-Bissau, Kazakstán, Líbano, Mada- } \\
\text { gascar, Malaysia, I. Mauricio, Mongolia, Mozambique, Ho- } \\
\text { landa, Nueva Zelanda, Nigeria, Rusia, Sierra Leona, Surinam, } \\
\text { Suiza, Tanzania, Togo }\end{array}$ \\
\hline
\end{tabular}

Se han obviado los países de población poco significativa ( $<50.000$ habitantes).

Fuente: elaboración propia. 
de 0 (peor) a 100 (mejor), se obtiene ponderando esos 25 indicadores en función de su importancia para conseguir unos objetivos idóneos señalados por los especialistas. Los indicadores, a su vez, se normalizan en función de la proximidad al objetivo idóneo en cada uno de ellos. El EPI se descompone en una serie de categorías de política ambiental, que se dividen en dos grandes grupos: salud ambiental y salud de los ecosistemas. Cada uno de esos dos grupos está dividido en varios aspectos — contaminación del aire, enfermedades, agua, biodiversidad, etc.—, que se concretan en 25 indicadores cuantitativos - acceso a agua corriente o sanitarios, contaminación urbana, emisiones de óxidos de nitrógeno, ozono, escasez de agua, cobertura forestal, etc.- Para el análisis global entre países, he seleccionado los valores del índice final, de cara a comparar si había diferencias significativas entre grupos de países de distinta religión mayoritaria. Posteriormente, he realizado un análisis más detallado, seleccionando países tipo de las principales tradiciones religiosas con niveles de desarrollo económico y población similares. Para este segundo análisis, he comparado los valores correspondientes a los indicadores base para el cálculo del EPI.

La figura 1 muestra un gráfico de cajas (boxplot) con la distribución de los valores del EPI para los grupos de países con tradiciones religiosas mayoritarias. El gráfico muestra la situación del valor mediano del EPI para cada grupo, así como la posición de los cuartiles inferior y superior —los límites de las cajas-. Este tipo de gráficos son comunes para representar variables no paramétricas (Tukey, 1977). La comparación entre los valores del EPI para países con distintas religiones no ofrece una clara conclusión, salvo la presencia de valores algo más bajos en países musulmanes. Las diferencias entre países budistas, cristianos e hindúes resultan poco significativas.

Entre los factores de control que los mismos autores del EPI proponen está el nivel de riqueza y desarrollo tecnológico, que afectan considerablemente a los indicadores ambientales. Como los mismos autores del índice sugieren, «el nivel de riqueza se correlaciona altamente con los indicadores del EPI, particularmente con los relacionados directamente con la salud» (Emerson et al., 2010 , p. 6). Sin embargo, esta relación no es válida para algunos países, que no ofrecen valores equiparables a otros con similar nivel de riqueza, lo que apunta la posibilidad de que haya otros factores relevantes, como la percepción de los ciudadanos, su actitud ante el medio ambiente o la situación política del país: «Esos análisis estadísticos sugieren que en muchos casos el nivel de gobernanza contribuye a mejores resultados ambientales» (Emerson et al., 2010 , p. 6). El impacto del nivel de riqueza sobre la calidad ambiental se evi- 
FIGURA 1

GRÁFICO DE CAJAS DE LOS VALORES DEL EPI POR GRUPOS RELIGIOSOS DE PAÍSES

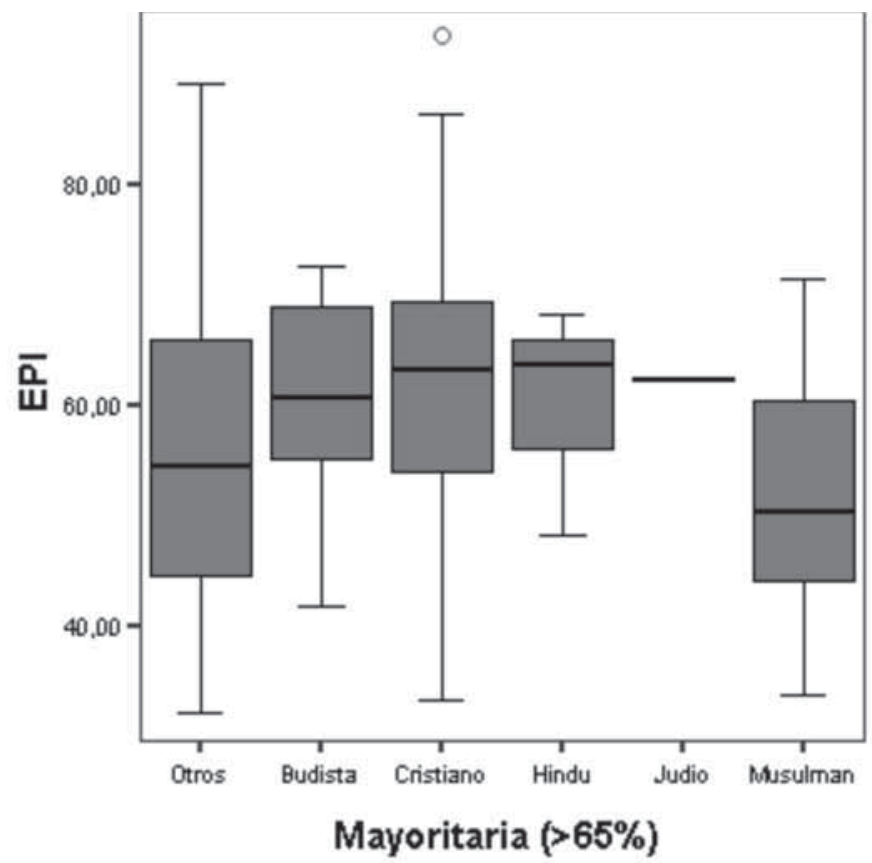

Para el judaísmo, no existe dispersión ya que sólo incluye un caso, el de Israel.

Fuente: elaboración propia.

dencia en un gráfico bivariado que muestre el valor del EPI y la renta per cápita (figura 2). En el gráfico se muestra una tendencia positiva en la correlación, pero con un valor explicativo bajo $\left(\mathrm{r}^{2}=0.37\right)$, lo que indica que hay bastante dispersión en esa correlación. En el gráfico se evidencia que las religiones no suponen una clara distinción en niveles de renta, ya que hay valores altos y bajos en cada una de ellas. En los valores más bajos del EPI aparecen países cristianos, musulmanes y no adscritos, si bien entre los más ricos, generalmente los valores del EPI son más bajos para los musulmanes, mientras los valores más altos se ocupan por cristianos y otros, tanto para valores medios como altos de renta per cápita. Para las rentas bajas ( $<1.000 \$ /$ hab), destaca el caso de Nepal, que tiene un valor de EPI mucho más alto $(68,2)$ que los demás países con similar nivel de renta. 
FIGURA 2

DIAGRAMA DE DISPERSIÓN ENTRE EPI Y RENTA PER CÁPITA (EN \$/HABITANTE) POR GRUPOS RELIGIOSOS DE PAÍSES

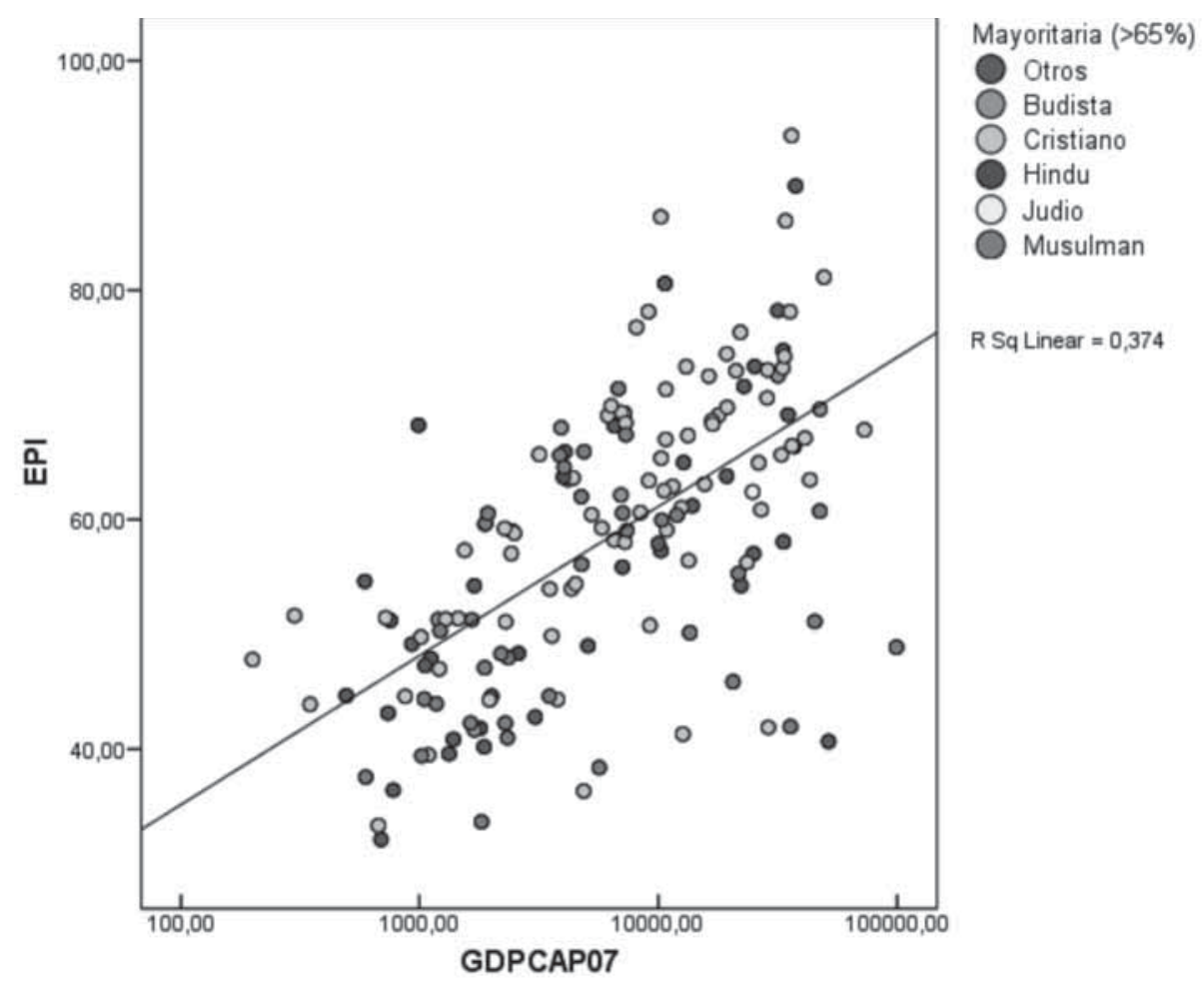

Fuente: elaboración propia.

Hemos realizado un análisis un poco más detallado de las relaciones entre EPI y el nivel de riqueza, calculando regresiones parciales entre ambas variables por grupos religiosos de países. Los resultados aparecen en la tabla 2 . Se muestra cómo entre budistas, cristianos y otros, la relación entre renta y EPI es clara y positiva. Esto quiere decir que para estos países el limitante de la calidad ambiental es la pobreza económica: los más ricos tienen mejores indicadores ambientales. El caso del budismo es especialmente claro por el peso de Japón y Singapur, ambos con niveles altos de EPI y muy altos de renta per cápita. Sin embargo, Bután consigue valores similares a Singapur —68 frente a 69,6 - con una renta más de doce veces inferior. Es llamativo el caso de los 
tres países hinduistas, donde se da una correlación negativa. El país más pobre, Nepal, tiene unos valores de calidad ambiental muy superiores a la India - 68,2 frente a 48,3 - , pese a tener una renta per cápita casi tres veces inferior. Sri Lanka tiene mayor renta que la India y valores EPI algo inferiores a Nepal. Otro elemento interesante a destacar es la práctica ausencia de correlación entre nivel de riqueza y EPI para los países musulmanes, lo que sugiere que los temas ambientales no son prioritarios en la consideración del desarrollo económico para buena parte de estos países.

TABLA 2

CORRELACIÓN Y COEFICIENTES DE REGRESIÓN ENTRE EL EPI Y EL LOGARITMO NATURAL DE LA RENTA PER CÁPITA

\begin{tabular}{lccr}
\hline & $\mathbf{r}^{2}$ & Pendiente & Constante \\
\hline Budistas & 0.609 & 5.7767 & 11.295 \\
Cristianos & 0.440 & 6.1198 & 7.359 \\
Hinduistas & 0.177 & -6.1439 & 107.324 \\
Musulmanes & 0.088 & 2.3743 & 31.885 \\
Otros & 0.497 & 6.3916 & 1.079 \\
\hline
\end{tabular}

Fuente: elaboración propia.

Para elaborar un análisis más detallado entre indicadores ambientales y tradiciones religiosas, hemos seleccionado países representativos de cada una de ellas, procurando que se tratara de casos con similar volumen de población y de renta. Hemos hecho dos comparaciones, una con países grandes y otra con países intermedios, pues entendemos que los temas ambientales también se afectan por el espacio que deben gestionar los distintos estados. En la tabla 3 se presentan los indicadores demográficos y territoriales de los países seleccionados. Es difícil que las comparaciones sean completamente fidedignas, ya que siempre hay diferencias en tamaños, población o renta, pero he intentado que las variaciones entre los países elegidos fueran las menores posibles. En cuanto a países emergentes, he seleccionado a Brasil, como ejemplo de país cristiano; a Indonesia, como país musulmán; a la India, como país hinduista y; a China, como país ateo-budista. En los tres primeros casos, la proporción de practicantes de cada religión es abrumadora, por lo que pueden considerarse ejemplos de las tradiciones religiosas que representan. Cuentan, además, con 
niveles de renta, población y territorio que los constituyen en potencias emergentes, aunque hay diferencias entre ellos, tanto en volumen de población, como en territorio o renta. En cuanto a la orientación religiosa, el caso de China es el más discutible, puesto que su adscripción religiosa es compleja. No es un país estrictamente budista, pero cuenta con raíces culturales claramente identificadas con esta tradición, por más que oficialmente sea un país ateo y el nivel de práctica religiosa no sea muy alto.

En cuanto a los países intermedios, se han seleccionado Túnez, Colombia, Tailandia, Sri Lanka e Israel. Salvo este último caso — que es imprescindible si queremos considerar el judaísmo-, los demás tienen valores de renta más o menos similares, con volúmenes de población y territorio intermedios entre los muy pequeños y las potencias anteriormente citadas.

TABLA 3

DATOS BÁSICOS DE LOS PAÍSES COMPARADOS

\begin{tabular}{|c|c|c|c|c|c|c|}
\hline País & PIB/CAP & Pob2007 & Área $\left(\mathrm{km}^{2}\right)$ & $\begin{array}{c}\text { Densidad } \\
\left(\mathrm{h} / \mathrm{km}^{2}\right)\end{array}$ & Religión & Practica \\
\hline Brasil & 9.146 & 190.119 .995 & 8.511 .044 & 22 & Cristianismo & $92 \%$ \\
\hline China & 5.084 & 1318.309.724 & 9.198 .094 & 143 & $\begin{array}{l}\text { Budismo } \\
\text { (ateísmo) }\end{array}$ & $20 \%$ \\
\hline Indonesia & 3.504 & 225.630 .065 & 1.897 .812 & 119 & Islam & $87 \%$ \\
\hline India & 2.600 & 1124.786 .997 & 3.208 .099 & 351 & Hinduismo & $80 \%$ \\
\hline Túnez & 7.102 & 10.225 .400 & 147.881 & 69 & Islam & $98 \%$ \\
\hline Colombia & 8.109 & 43.987 .000 & 1.141 .177 & 39 & Cristianismo & $94 \%$ \\
\hline Tailandia & 6.983 & 66.979 .359 & 513.635 & 130 & Budismo & $95 \%$ \\
\hline Sri Lanka & 4.007 & 20.010 .000 & 65.830 & 301 & Hinduismo & $70 \%$ \\
\hline Israel & 24.824 & 7.180 .100 & 21.878 & 328 & Judaísmo & $78 \%$ \\
\hline
\end{tabular}

Las primeras filas para los emergentes, y las siguientes para los intermedios.

Fuente: elaboración propia.

De los 25 indicadores cuantitativos que forman el EPI, para simplificar un poco los análisis, hemos seleccionado 10 para las comparaciones, además de los dos índices sintéticos del EPI — Salud ambiental y de los ecosistemas-. Estos 10 indicadores resumen bien, a nuestro juicio, los 25 iniciales y repre- 
sentan los 10 campos de interés ambiental que definen los autores del EPI. Se trata de los siguientes:

- AAT, porcentaje de viviendas con sanitarios, aguas tratadas;

- PA, proporción de partículas en el aire en zonas urbanas;

- $\mathrm{SO}_{2}$, emisiones de dióxido de sulfuro por área habitada;

- ICA, calidad del agua calculada por UNEP, que incluye contenido en oxígeno, nitrógeno, fósforo y conductividad eléctrica;

- UD, índice de uso/disponibilidad de agua, que relaciona la cantidad de agua que se usa sobre la que se dispone, e incluye desalinización, agua subterránea renovable y no;

- PA, proporción de hábitats críticos que están protegidos;

- VC, variación de cobertura arbolada entre 2005 y 2000 tal y como lo reporta la FAO;

- PS, pesca sostenible, definida como la proporción de la pesca que se hace en los estratos más bajos de la cadena alimentaria;

- IR, intensidad del regadío, definida como el agua empleada para agricultura, frente a la producción obtenida;

- EG, eficiencia en la generación eléctrica, calculada a partir de las emisiones de $\mathrm{CO}_{2}$ a consecuencia de la generación de energía.

En la tabla 4 se recogen los valores normalizados de estos índices para los cuatro países que hemos seleccionado como representativos de las grandes religiones. Los autores del EPI normalizan el valor de cada índice entre 0 y 100 en función de la cercanía a los objetivos idóneos para cada indicador, de cara a poder relacionarlos entre sí como índices sintéticos (Emerson et al., 2010). Si consideramos como situación razonablemente buena tener un valor por encima de 70 y como mala por debajo de 35, la situación del país cristiano (Brasil) es muy superior al resto, con 7 indicadores en situación buena y sólo 1 en situación mala, frente a 4 y 3, respectivamente, para el país budista (China), 3 y 3 para el musulmán (Indonesia), y 3 y 3 para el hinduista (India).

En el caso de los países intermedios, observamos tendencias similares (tabla 5). El país cristiano (Colombia) ofrece las mejores tendencias, con 6 indicadores en situación buena y ninguno en situación mala. El país musulmán (Túnez) sobre 9 indicadores — no hay datos de protección de hábitatsofrece 5 con niveles buenos y 1 con malos; mientras el budista (Tailandia) tiene 6 sobre 9 en buenos y 2 en malos, y el hinduista (Sri Lanka) tiene 5 sobre 10 buenos y 2 malos. Finalmente el caso del país judío (Israel) es llamativo, pues pese a tener la renta más alta de estos países intermedios — casi 4 veces superior-, ofrece indicadores ambientales poco idóneos, con solo 5 en 


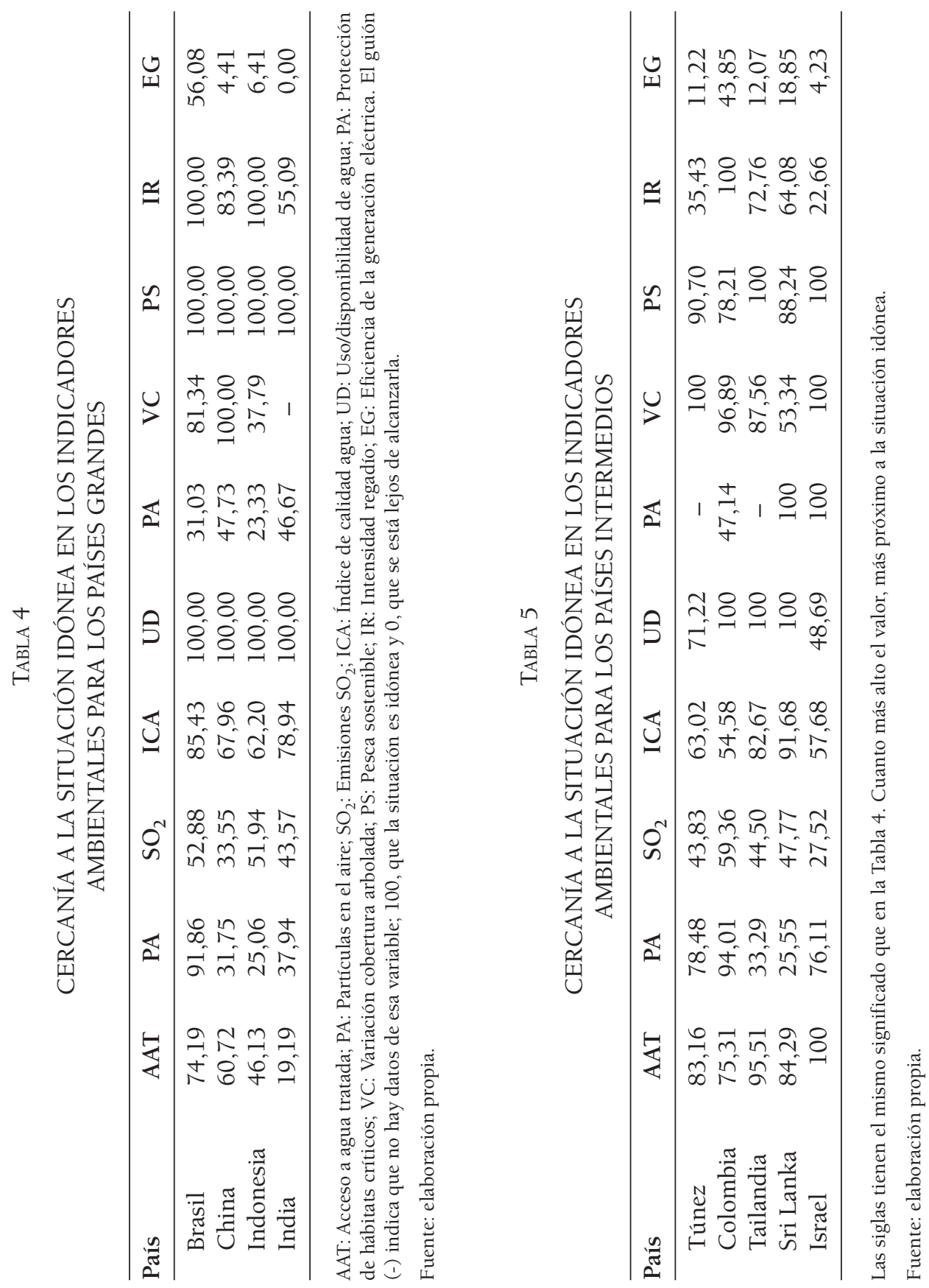

Estudios Geográficos, Vol. LXXIII, 273, pp. 421-447, julio-diciembre 2012 ISSN: 0014-1496, eISSN: 1988-8546, doi: 10.3989/estgeogr.201215 
situación buena y 3 en situación mala, alcanzando incluso las peores puntuaciones de los países seleccionados en dos de los indicadores.

Si se consideran los dos índices sintéticos que forman el EPI — salud ambiental y salud de los ecosistemas- se refuerzan los comentarios antes realizados (tabla 6). El primero ofrece el valor más alto para Israel, que paradójicamente tiene la peor puntuación en el índice de salud de los ecosistemas, ofreciendo un valor final intermedio, pese a tratarse del país con la renta per cápita más alta entre los analizados. Le sigue Túnez y los dos países cristianos (Colombia y Brasil). Más alejados están los países budistas (Tailandia y China) y el escalón más bajo lo ocupan los dos hinduistas (India y Sri Lanka), junto a Indonesia, que tiene el segundo valor más bajo. En cuanto al indicador de salud de los ecosistemas, las tendencias son divergentes, con el valor más alto para Sri Lanka y Colombia, seguidos a distancia por Tailandia y Brasil. Respecto al índice final EPI, los países cristianos ofrece los valores más altos - primer y tercer puesto-, mientras que los musulmanes se sitúan en los valores más bajos del ranking — sexto y noveno-, y el resto de las religiones ofrecen valores intermedios: budistas — quinto y séptimo—, hinduistas —-segundo y octavo-, y el Estado de Israel ocupa el cuarto puesto.

TABLA 6

ÍNDICES INTEGRADOS DEL EPI E ÍNDICE FINAL

\begin{tabular}{lccc}
\hline País & Salud Ambiental & Salud Ecosistemas & EPI final (ranking) \\
\hline Túnez & 77,16 & 44,00 & $60,58\left(6^{\circ}\right)$ \\
Colombia & 74,60 & 78,94 & $76,77\left(1^{\circ}\right)$ \\
Tailandia & 65,58 & 58,73 & $62,15\left(5^{\circ}\right)$ \\
Sri Lanka & 45,84 & 81,59 & $63,71\left(2^{\circ}\right)$ \\
Israel & 92,11 & 32,75 & $62,43\left(4^{\circ}\right)$ \\
Brasil & 71,63 & 55,19 & $63,41\left(3^{\circ}\right)$ \\
China & 58,68 & 39,33 & $49,00\left(7^{\circ}\right)$ \\
Indonesia & 44,59 & 44,64 & $44,61\left(9^{\circ}\right)$ \\
India & 41,59 & 55,10 & $48,35\left(8^{\circ}\right)$ \\
\hline
\end{tabular}

Fuente: elaboración propia. 


\section{DISCUSIÓN Y CONCLUSIONES PRELIMINARES}

A partir de los indicadores de calidad ambiental que recoge el EPI, hemos presentado una primera evaluación de las condiciones ambientales para grupos de países en función de su confesión religiosa mayoritaria. Con las limitaciones propias del análisis, hemos visto que los países cristianos no sólo no tienen valores ambientales peores que otros, sino más bien al contrario, tanto en el global como en los casos seleccionados de países grandes-emergentes y medios. Sería aventurado concluir que las diferencias se deben a que el cristianismo sea una religión más conservacionista que el islam o el budismo, pero al menos podemos rechazar la hipótesis contraria.

Ciertamente la visión religiosa de las personas no es el único elemento a considerar, ni siquiera tal vez el más importante, para explicar el estado de conservación ambiental de un determinado territorio. Por esa razón, hemos intentado sectorizar el análisis por grupos de países con similares niveles económicos o magnitudes territoriales. Aún así, sigue sin confirmarse que el cristianismo genere países con peores indicadores ambientales.

Podría plantearse como explicación de los datos observados que las religiones tienen poco peso actualmente para conformar valores sociales y, que por tanto clasificar los países en función de su religión mayoritaria es irrelevante para explicar una determinada variable ambiental. De hecho, en los datos presentados, se observa que esto no es así, ya que hay diferencias notables entre las tendencias de un grupo de países y otros. Se podrían señalar múltiples diferencias sociales entre países con distinta mayoría religiosa, por ejemplo en lo que se refiere a la alimentación, las fiestas laborales, o la reglamentación familiar. Es bien sabido que algunas religiones prohíben el consumo de alcohol — singularmente el islam-. Si para estas religiones, esa actuación es relevante, parecería razonable encontrar distintos niveles de consumo de alcohol, por ejemplo en unos países mayoritariamente islámicos frente a otros que no reglamenten ese consumo. Los datos más recientes de la "Organización Internacional de la Viña y el Vino" (OIV), confirman esta hipótesis, pues los países musulmanes de los que se dispone de datos tienen un consumo promedio más de diez veces inferior al de los cristianos - 1,5 litros por persona y año, frente a $16,4^{4}$ - , siendo significativamente más bajo $(\mathrm{p}<0.01)$. Es un ejemplo claro en donde la práctica religiosa tiene claras consecuencias sociales.

\footnotetext{
4 Datos tomados de: http://www.oiv.int/oiv/info/enstatoivextracts2 (Fecha de consulta: agosto 2012).
} 
Volviendo al tema principal de este artículo, si no encontramos peores indicadores ambientales en los países cristianos, habríamos de concluir que o bien el cristianismo no es en realidad tan agresivo con el medio como se le ha acusado, o bien que otras religiones más conservacionistas - como hinduismo o budismo- no han establecido prioridades muy claras en sus políticas de desarrollo. Hemos visto en este trabajo que, pese al interés del artículo de White, éste contiene varias simplificaciones teológicas e históricas, y que dentro del cristianismo hay tradiciones mucho más amistosas con el medio, que parecen haber sido más protagonistas que sus contrarias para definir políticas de desarrollo.

Como ya hemos indicado, hay otros factores relevantes para explicar la calidad ambiental, como el acceso a la tecnología o el nivel de riqueza. Con los datos presentados, hemos visto que el nivel de renta está directamente relacionado con el estado de conservación ambiental para los países cristianos y budistas, pero no tanto para los musulmanes e hinduistas, en donde incluso se dan relaciones negativas. El judaísmo no sale tampoco bien parado en el análisis, ya que ofrece indicadores de calidad ambiental muy por debajo de lo que cabría esperar por su nivel de riqueza. En pocas palabras, en algunos grupos de países se observa que el limitante de la conservación no es la cultura - religión- sino la técnica-economía — renta-, mientras en otros, incluso con niveles de renta altos, la conservación ambiental no parece ser una prioridad. La afirmación sería más claramente atribuible a algunos países musulmanes, que cuentan con muy alta renta per cápita junto a valores medios y bajos del EPI. En este sentido, se confirmarían algunas críticas a las prioridades ambientales de los países islámicos que han hecho algunos pensadores musulmanes: «Cuando uno estudia la visión islámica de la naturaleza y de las relaciones de la sociedad con el medio ambiente, cuando se analiza la forma en que la civilización islámica clásica creó una sociedad, y especialmente un ambiente urbano, en armonía con la naturaleza, y se compara con la situación actual del mundo islámico, resulta obvio concluir que ni los gobiernos musulmanes, ni la mayor parte de sus gentes están siguiendo los principios islámicos en su trato con el medio ambiente natural» (Nasr, 2003, p. 87).

Estas afirmaciones son especialmente pertinentes para los países musulmanes, que incluso cuentan con una legislación religiosa que rige buena parte de la vida social sin que esto implique consecuencias ambientales claras. No es el caso del cristianismo, que nunca ha pretendido generar teocracias, pero ciertamente ha tenido y tiene un importante papel en generar valores éticos y formas de actuar.

En cualquier caso, es importante subrayar la importancia de que las grandes religiones hagan más vivo su compromiso con la conservación ambiental, 
principalmente a través de reforzar la formación ambiental de sus líderes. En este sentido, se hace cada vez más viva la necesidad de profundizar en las implicaciones ambientales de las distintas tradiciones religiosas, de tal manera que, de cara al futuro, sean mejores aliados en el esfuerzo colectivo por mejorar nuestra relación con la naturaleza, conduciéndonos a un uso más sustentable de los recursos del planeta.

Fecha de recepción: 31/08/2012

Fecha de aceptación: 16/10/2012

\section{BiBLIOGRAFÍA}

Berry, R. J. (ed.) (2006): Environmental Stewardship. London, T\&T Clark International. Berry, T. (1988): The Dream of the earth. San Francisco, Sierra Club.

Chuvieco, E. (2012a): "¿Qué aportan las grandes religiones a la conservación ambiental?", en E. Chuvieco y D. Alexander, D. (eds.): Ciencia y Religión en el siglo xxI: recuperar el diálogo. Madrid, Editorial Centro de Estudios Ramón Areces, pp. 239-268.

Chuvieco, E. (2012b): "Religious approaches to water management and environmental conservation". Water Policy, 14, pp. 9-20.

Chuvieco, E. y Martin, M. A. (2012): ¡Dominad la Tierra!: Raíces filosóficas y teólogicas del ecologismo. Madrid, Digital Reasons (www.digitalreasons.es).

Emerson, J.; Esty, D. C.; Levy, M. A.; Kim, C. H.; Mara, V.; Sherbinin, A. D. y Srebotnjak, T. (2010): Environmental Performance Index. New Haven, Yale Center for Environmental Law and Policy. Disponible en: http://epi.yale.edu/ (Fecha de consulta: 03/09/2012).

Foltz, R. C.; Denny, F. M. y Baharuddin, A. (eds.) (2003): Islam and Ecology. A Bestowed Trust. Cambridge, MA, Harvard University Press.

Harrison, P. (2006): "Having Dominion: Genesis and the Mastery of Nature", en: Berry, R. J. (ed.): Environmental Stewardship. London, T\&T Clark International, pp. 17-31.

Hessel, D. T. y Ruether, R. R. (eds.) (2000): Christianity and Ecology: Seeking the Wellbeing of Earth and Humans. Cambridge, MA, Harvard University Press.

Houghton, J. (2006): "Stewardship for the Twenty-first Century", en R. J. Berry (ed.): Environmental Stewardship. London, T\&T Clark International.

McDonagh, S. (1986): To care for the earth: a call to a new theology. London, Geoffrey Chapman.

Nasr, S. H. (2003): "Islam, the Contemporary Islamic world, and the Environmental Crisis", en R. Folz; F. Denny y A. Baharuddin (eds): Islam and Ecology. Cambridge, MA, Harvard University Press, pp. 85-105.

Palmer, C. (2006): "Stewardship: A Case study in Environmental Ethics", en R. J. Berry, (ed): Environmental Stewardship. London, T\&T Clark International, pp. 63-75.

San Agustin (413-426): La Ciudad de Dios. Madrid, BAC 171-172, 1958, pp. lib 22, cap 24.

San Francisco (1225): Cantico del hermano Sol. Diponible en: http://www.franciscanos.org/esfa/cant.html (Fecha de consulta: 03/09/2012). 
Sorrell, R. D. (1988): St. Francis of Assisi and nature: Tradition and innovation in Western Christian attitudes toward the environment. New York, Oxford, Oxford University Press.

Sotelo, J. A.; Tolón, A. y Lastra, X. (2011): "Indicadores por y para el desarrollo sostenible, un estudio de caso". Estudios Geográficos, 72/271, pp. 611-654.

Tirosh-Samuelson, H. (ed.) (2002): Judaism and Ecology. Cambridge, MA, Harvard University Press.

Tucker, M. E. y Grim, J. (2003): "Series Foreword”, en R. Folz, F. Denny y A. Baharuddin (eds.): Islam and Ecology. Cambridge, MA, Harvard University Press, p. xvi.

Tucker, M. E. y Williams, D. R. (eds.) (1997): Buddhism and Ecology: The Interconnection of Dharma and Deed. Cambridge, MA, Harvard University Press.

Tukey, J. W. (1977): Exploratory Data Analysis. Reading, MA, Addison-Wesley.

White, L. (1967): "The Historical Roots of Our Ecological Crisis". Science, 155/3767, pp. 1203-1207.

Woods, T. E. (2005): How the Catholic Church built Western Civilization. Washington, D.C., Regnery Publications Inc.

\section{RESUMEN}

En los años sesenta se inicia el debate sobre el papel que el cristianismo ha tenido en la degradación ambiental del planeta, acusándosele de haber concedido al ser humano un estatus dominante frente al resto de los seres creados. En este artículo se revisan las distintas respuestas que se han dado a estas críticas desde el punto de vista histórico y teológico. En la segunda parte, se analiza hasta qué punto este supuesto carácter agresivo del cristianismo frente a otras religiones se manifiesta en la situación ambiental de los países cristianos frente a otros donde son mayoritarias otras religiones. Para ello, se emplean distintos indicadores relacionados con la salud ambiental y la salud de los ecosistemas. Se concluye que los países cristianos no presentan una peor situación ambiental que los representativos de otras religiones. Se observa una correlación clara entre nivel de riqueza y de calidad ambiental para los países cristianos, que no es tan evidente en los hindúes o musulmanes. A similar nivel de riqueza y población, los países cristianos de hecho ofrecen valores más altos de calidad ambiental que los encontrados en países con otras tradiciones religiosas. Si bien de nuestro análisis no puede concluirse que el cristianismo promueva la conservación ambiental, los resultados indican al menos que puede rechazarse la hipótesis contraria, ya que los países cristianos no presentan indicadores significativamente peores que los encontrados en países representativos de otras tradiciones religiosas.

PALABRAS ClaVE: cristianismo; conservación ambiental; ética ambiental.

\section{Abstract}

As a result of a paper published in the sixties, Christianity has been frequently accused of being behind earth's environmental degradation, on the grounds that it has given humans the religious support to adopt a dominant role over the rest of Crea- 
tion. This paper explores some of the answers to those criticisms, both from a historical and theological point of view. In the second part, the paper explores to what extent the environmental degradation supposedly caused by Christianity has affected those countries with mostly Christian populations, comparing them with those with other religious traditions. Several indicators of environmental health and ecosystem conservation are used for the comparisons. A high correlation between per capita income and environmental performance for the Christian and Buddhist countries was observed, while poor trends were observed for Hindu and Islamic countries. Comparing representative countries of both large and medium population size, the Christian countries outperform others in most indicators. Even though it cannot be concluded that Christianity, in fact, encourages environmental conservation, the results from our analysis at least provide grounds to reject the opposite hypothesis, as Christian countries do not have significantly lower environmental indicators than those representing other major religions.

KEY WORDS: Christianity; environment; conservation; ethics.

\section{RÉSUMÉ}

Les années soixante ont assisté à la naissance du débat sur le rôle joué par le christianisme dans la dégradation environnementale de la planète, étant accusé d'avoir donné à l'espèce humaine un statut dominant par rapport aux autres créatures. Cet article révise les différentes réponses données à ces critiques du point de vue historique et théologique. La deuxième partie analyse à quel point ce caractère soi-disant agressif du christianisme face à d'autres religions se manifeste dans la situation environnementale des pays chrétiens comparés à d'autres dans lesquels les religions majoritaires sont différentes. Pour cela, des indicateurs divers sont employés concernant la santé environnementale et la santé des écosystèmes. La conclusion obtenue est que les pays chrétiens ne présentent pas une situation environnementale pire que ceux représentatifs d'autres religions. Une corrélation évidente est observée entre le niveau de richesse et la qualité environnementale pour les pays chrétiens, pas aussi évidente pour les hindous ou les musulmans. Avec un niveau semblable de richesse et de population, les pays chrétiens présentent en fait des valeurs plus élevées de qualité environnementale que celles trouvées dans les pays avec d'autres traditions religieuses. Bien que notre analyse ne permette pas de conclure que le christianisme encourage la protection environnementale, les résultats indiquent au moins que l'hypothèse contraire peut être refusée, puisque les pays chrétiens ne présentent pas d'indicateurs significativement plus négatifs que ceux trouvés en des pays représentatifs d'autres traditions religieuses.

MOTS CLÉS: christianisme; protection environnementale; éthique environnementale. 